\title{
25 Research Suare \\ Serum uromodulin is a novel predictive marker of renal function in the Japanese population
}

Ryosuke Usui ( $\square$ usui.ryosuke@twmu.ac.jp )

Yachiyo Medical Center, Tokyo Women's Medical University

Tetsuya Ogawa

Tokyo Women's Medical University, Medical Center East

Hideo Takahashi

Minami Akatsuka Clinic

Chihiro Iwasaki

Yachiyo Medical Center, Tokyo Women's Medical University

Minako Koike

Yachiyo Medical Center, Tokyo Women's Medical University

Taku Morito

Yokohama Rosai Hospital

Michiyasu Hatano

Yokohama Rosai Hospital

Kosaku Nitta

Tokyo Women's Medical University

\section{Research article}

Keywords: uromodulin, Tamm-Horsfall protein, kidney function, CKD, biomarker

Posted Date: March 23rd, 2020

DOI: https://doi.org/10.21203/rs.3.rs-18644/v1

License: (c) (1) This work is licensed under a Creative Commons Attribution 4.0 International License.

Read Full License 


\section{Abstract}

Background Uromodulin, also known as Tamm-Horsfall protein, is the most abundant protein in urine. It has recently been reported that uromodulin exists in a small amount in blood and that its concentration correlates with the estimated glomerular filtration rate (eGFR). However, its clinical significance has not been clarified and there are no relevant data for the Japanese population.

Method First, we generated anti-human uromodulin mouse monoclonal antibodies and established a specific enzyme-linked immunosorbent assay for uromodulin. We then performed an observational clinical study to determine if there was a correlation between serum uromodulin concentration and estimates of kidney function and whether the serum uromodulin value could be a biomarker in clinical nephrology. The clinical study included 308 patients with and without chronic kidney disease and healthy volunteers. Serum concentrations of creatinine, cystatin C, and uromodulin were measured and correlations were sought between the eGFR calculated from the creatinine and cystatin $\mathrm{C}$ levels and the serum uromodulin concentration.

Results There was a good correlation between the serum uromodulin concentration and the eGFR value calculated from the creatinine $(r=0.76)$ and cystatin $C(r=0.79)$ levels. The mean serum uromodulin level in the group with an eGFR $>90 \mathrm{~mL} / \mathrm{min} / 1.73 \mathrm{~m} 2$ calculated using cystatin $\mathrm{C}$ was significantly higher than that in the group with an eGFR of $80-89 \mathrm{~mL} / \mathrm{min} / 1.73 \mathrm{~m} 2$.

Conclusions The serum uromodulin measurement could be a useful biomarker for identification of patients with early deterioration of kidney function.

\section{Background}

Uromodulin, also known as Tamm-Horsfall protein, is isolated as a highly glycosylated mucoprotein that inhibits viral hemagglutination, is exclusively expressed in the kidney, and is the most abundant protein in urine [1]. Almost all of the uromodulin in the kidney is released from the luminal surface of tubular epithelial cells between the thick ascending limb of Henle's loop and the early distal convoluted tubule. Uromodulin undergoes proteolytic cleavage and is excreted in the urine.

In 2003, the American Heart Association formally recognized chronic kidney disease (CKD) as a strong risk factor for cardiovascular disease [2], and the importance of therapeutic intervention for CKD as early as possible is now well understood. Unfortunately, it is not realistic to treat such a large number of patients, and the focus should be on identifying patients with CKD who are at higher risk for cardiovascular disease. However, the conventional kidney biomarkers, such as creatinine, cystatin C, and urinary albumin, have some limitations when used for screening purposes.

A small amount of uromodulin has recently been detected in blood and its concentration correlates well with estimated glomerular filtration rate (eGFR) values calculated from creatinine and cystatin C [3-5]. In the past decade, several genome-wide association studies have identified common variants in the 
promoter region of the UMOD gene as risk factors for progression to CKD and hypertension [6-9]. It has also been reported that the serum uromodulin concentration correlates with mortality in patients with cardiovascular disease $[10,11]$. Moreover, the findings of in vivo studies in an experimental mouse model lacking the uromodulin gene suggest that uromodulin has a renoprotective effect [12,13]. These reports suggest that uromodulin might be a useful renal biomarker. However, as yet, there are no data on serum uromodulin measurements in the Japanese population.

On the other hands, now we can use some commercially available enzyme-linked immunosorbent assay (ELISA) kits for measuring blood uromodulin, but the antibody information used in the ELISA kits is not disclosed. As described above, blood uromodulin is considered to have great potential as a novel renal biomarker. Therefore, we generated original monoclonal antibodies, investigated and disclosed antibody information including theirs antigen epitopes, established the custom-made ELISA for serum uromodulin, and performed a clinical study to evaluate the importance and usefulness of serum uromodulin measurement in clinical nephrology.

\section{Methods}

\section{Preclinical study}

We generated monoclonal antibodies (mAbs) against human uromodulin protein using the standard method and established an ELISA for measurement of the serum uromodulin concentration. Generation of mAbs and creation of the ELISA were performed as a joint research and development project between Tokyo Women's Medical University and Immuno-Probe Co., Ltd (Saitama, Japan).

The procedures for the basic experiments, such as generation of mAbs, creation of the ELISA, accuracy evaluation of ELISA, cloning of uromodulin gene, preparation of the expression vectors, cell culture, transfection, electrophoresis, Western blotting, animal breeding and uromodulin measurement, are described in supplementary information.

\section{Clinical study}

Participants, definition of CKD, and calculation of eGFR

This multicenter observational study was approved by the ethics committees of each participating medical institution and conducted in adherence with the Declaration of Helsinki. We enrolled 308 Japanese participants, including patients with and without CKD and healthy volunteers, who were 18 years of age or older. All participants attended each participating institution regularly over a period of 3 months and gave written informed consent before collection of demographic and clinical data and serum samples. At the time of the first sample collection, we confirmed that the variation in serum creatinine concentration was within $10 \%$ of the previous value. The exclusion criteria were as follows: refusal to participate in the study, non-Japanese ethnicity, acute illness, such as acute kidney injury or viral/bacterial infection within the previous 3 months, a diagnosis of cancer, renal transplantation, and 
pregnancy. CKD was defined as a GFR $<60 \mathrm{~mL} / \mathrm{min} / 1.73 \mathrm{~m}^{2}$ or abnormal urinary or imaging findings suggestive of a kidney disorder that had persisted for more than 3 months. The eGFR values were calculated based on age, sex, and serum creatinine [14] and cystatin C [15] concentrations using equations that are established for the Japanese population.

\section{Sample collection and uromodulin measurement}

The serum samples were collected immediately after blood collection, aliquoted and frozen until analysis. Procedure of uromodulin measuring using the custom-made ELISA is described in supplementary information. All uromodulin measurements were performed in the laboratory at Yachiyo Medical Center, Tokyo Women's Medical University.

\section{Statistical analysis}

All statistical analyses were performed using JMP Pro 14.0.0 software (SAS Institute Inc., Cary, NC). Continuous data are presented as the mean (with the standard deviation or standard error of the mean) or the median (with the interquartile range). The distribution of continuous variables was evaluated for normality using the Shapiro-Wilk test. Differences in means for adjacent groups were analyzed using the Student's $t$-test. Pearson correlation coefficient and Spearman's rank correlation analyses were used to evaluate the statistical significance of the relationship between two continuous variables, such as serum uromodulin concentration and eGFR. The tests were two-tailed and the results were considered statistically significant at a $P$-value of $<0.05$. Kruskal-Wallis analysis was used to compare multiple groups.

\section{Results}

\section{Preclinical study}

\section{Antibodies and their characteristics}

The Coomassie Brilliant Blue-stained purified mAbs separated by SDS-PAGE are shown in Supplementary Figure S1. Two bands, namely, heavy chain (50 kDa) and light chain ( $25 \mathrm{kDa})$, were observed in the reducing condition, whereas a single band of full-length immunoglobulin was observed in the nonreducing condition (Supplementary Figure S1A). No extra bands were seen. The detection sensitivity of each mAb was evaluated by Western blotting (Supplementary Figure S1B-C). Both antibodies had detection sensitivity that was several-fold greater for non-reduced samples than for reduced samples.

\section{Epitopes recognized by $m A b s$}

The 3-5 mAb could detect protein translated from N201-GFP and N222-GFP vectors but not from the N162-GFP vector (Supplementary Figure S3A). The epitope recognized by 3-5 mAb was the amino acids translated between nucleotide numbers 163 and 201 from the N-terminus (i.e., GFTGDGLTCVDLD). The 29-9 mAbs could detect protein translated by the N321-FLAG and N381-FLAG vectors but not by the 
N261-FLAG vector (Supplementary Figure S3B). The epitope recognized by 29-9 mAb was the amino acids translated between nucleotide numbers 262 and 321 from the N-terminus (i.e., GSFSCVCPEGFRLSPGLGCT). The location of the epitopes recognized by the 3-5 and 29-9 mAbs with respect to the full-length uromodulin protein are described in Supplementary Figure S3C.

Accuracy of ELISA, stability of uromodulin protein, and differences between serum and plasma measurements

A dilution test using standard protein confirmed acceptable linearity ( $r=0.99$, Supplementary Figure S4). The intra- and inter-assay coefficients of variation for the custom-made ELISA were $2.1-2.9 \%$ and $2.6 \%$, respectively (Supplementary Table S1A-C). The stability of serum uromodulin protein was then evaluated under the conditions described in the legend of Supplementary Table S2. These data suggest that uromodulin protein is very stable in serum at $4^{\circ} \mathrm{C}$ and that the stability of uromodulin protein is not dependent on its concentration (Supplementary Table S2). Furthermore, the uromodulin concentrations in serum samples were higher than those in plasma samples (Supplementary Table S3).

\section{Correlation between serum uromodulin concentration and eGFR}

The characteristics of the study participants are shown by sex in Table 1. Although there were significant differences in all parameters when classified by sex, high and same-leveled associations were confirmed by Pearson correlation coefficient and Spearman's rank correlation analyses in all groups (Figure 1A-F). The serum uromodulin concentration was shown to have a positive correlation with the eGFR on linear regression.

Serum uromodulin concentrations in patients with and without CKD and in healthy volunteers

Figure 2A shows a box plot of the serum uromodulin concentration divided with the creatinine-calculated eGFR (eGFR-cre). The mean serum uromodulin concentration was $274.1 \mathrm{ng} / \mathrm{mL}$ in patients without CKD and healthy volunteers, $265.0 \mathrm{ng} / \mathrm{mL}$ in patients with CKD stage $1,243.8 \mathrm{ng} / \mathrm{mL}$ in those with CKD stage $2,173.0 \mathrm{ng} / \mathrm{mL}$ in those with CKD stage $3 a, 120.5 \mathrm{ng} / \mathrm{mL}$ in those with CKD stage $3 b, 58.2 \mathrm{ng} / \mathrm{mL}$ in those with CKD stage $4,34.6 \mathrm{ng} / \mathrm{mL}$ in those with CKD stage $5,23.1 \mathrm{ng} / \mathrm{mL}$ at the time of hemodialysis introduction, and $4.7 \mathrm{ng} / \mathrm{mL}$ in patients with CKD stage 5D. Among the adjacent groups, there was a significant difference in the mean serum uromodulin concentration between CKD stage 2 and CKD stage $3 a$ and between CKD stage $3 a$ and CKD stage $3 \mathrm{~b}$.

A box plot of the serum uromodulin concentration divided with the cystatin C-calculated eGFR (eGFR-cys) is shown in Figure 2B. There was no significant difference in the mean serum uromodulin concentration between the patients with CKD stage 1 and the healthy volunteers and patients without CKD; however, the mean serum uromodulin concentration in patients with CKD stage 2 was significantly lower than that in patients with CKD stage 1. Moreover, the mean serum uromodulin concentration in the patients with CKD and an eGFR-cys of $81-90 \mathrm{~mL} / \mathrm{min} / 1.73 \mathrm{~m}^{2}$ was significantly lower than that in the patients with CKD and an eGFR-cys of $>90 \mathrm{~mL} / \mathrm{min} / 1.73 \mathrm{~m}^{2}$, and in healthy volunteers and patients without CKD. 


\section{Discussion}

To our knowledge, this is the first study of uromodulin concentrations undertaken in Japanese patients with chronic kidney disease. The results of this study are consistent with previous reports of a strong correlation between the serum uromodulin concentration and kidney function [3-5] and suggest that the serum uromodulin could be a unique biomarker in clinical nephrology. It had been thought that uromodulin is expressed only in renal tubules and that almost all of it is excreted in urine after undergoing proteolytic cleavage. However, in 1981, Dawnay et al. investigated blood uromodulin concentrations and identified trends between the serum uromodulin concentration and kidney disease, such as a level in the range of $70-540 \mathrm{ng} / \mathrm{mL}$ in patients with normal kidney function and undetectable serum uromodulin in patients who are anephric [16]. The first report of a good correlation between blood uromodulin concentration and kidney function was published by Thornley et al. in 1985 [17]. However, since then, there have been no detailed studies on blood uromodulin levels. In 2010, Prajczer et al. investigated the relationship between the serum uromodulin concentration and kidney function in healthy volunteers and CKD patients and did not find a significant correlation [18]. Although the authors analyzed serum samples using a custom-made ELISA, those concentrations were extremely low compared with others $[3-5,17]$. It is possible that they were not fully evaluated. However, Steubl et al. [3] and Fedak et al. [4] in 2016 and Scherberich et al. [5] in 2018 reported a good correlation between the plasma/serum uromodulin concentration and kidney function using commercially available ELISA kits manufactured by BioVendor (Brno, Czech Republic) and Euroimmun AG (Lübeck, Germany).

We started by obtaining monoclonal antibodies de novo and then established a custom-made ELISA kit. This kit was prepared as a sandwich-type ELISA using two types of monoclonal antibodies recognizing different antigenic epitopes. As a preanalytical investigation, we confirmed that the accuracy of the kit was acceptable. Subsequently, we confirmed that the uromodulin protein was stable with no change in the measured value despite some variation in storage conditions; however, blood samples were handled suitably in this study. Scherberich et al. also reported the stability of uromodulin protein [5]. Stability is a very important issue when considering a protein for use as a biomarker. If the entity to be measured decomposes easily, its clinical application is not realistic. In this regard, uromodulin meets one of the requirements for use as a biomarker. Next, the uromodulin concentration was found to vary between serum and plasma samples. Previous studies have evaluated serum [4,5] and plasma samples [3] and both have shown a good correlation between blood uromodulin and GFR. In this study, we only analyzed serum samples.

Kidney function is indicated by the GFR, accurate measurement of which is best achieved by the inulin clearance test. However, this test is rarely performed because of its complexity. Kidney function has recently been evaluated by eGFR values calculated from creatinine and/or cystatin C. Although equations from the Modification of Diet in Renal Disease Study [19] and the Chronic Kidney Disease Epidemiology Collaboration [20] have been used internationally, they tend to overestimate kidney function in the Japanese population. Therefore, we Japanese should use the eGFR equations established for Japanese population $[14,15]$. eGFR is convenient for clinical use but not without problems. Given that eGFR is 
calculated from creatinine and/or cystatin $\mathrm{C}$, its value is affected by numerical fluctuations in these parameters, which may not necessarily be directly related to kidney function. It has been shown that the creatinine concentration is affected by muscle mass and fluid volume and that the cystatin $\mathrm{C}$ concentration is affected by thyroid function [21] and several drugs [22]. Kazama et al. reported a $72 \%$ positive rate below $50 \mathrm{~mL} / \mathrm{min}$ of GFR by creatinine measurement and an $85 \%$ positive rate below 70 $\mathrm{mL} / \mathrm{min}$ of GFR by cystatin $\mathrm{C}$ measurement when assessing the detection power of decreased kidney function [23]. Therefore, cystatin $\mathrm{C}$ is more useful than creatinine for detecting an early decline in kidney function. In contrast, it is known that cystatin $C$, unlike creatinine, has a ceiling effect at about 5-6 mg/L, and if it exceeds $2 \mathrm{mg} / \mathrm{L}$, the clinical significance of estimating kidney function by cystatin $\mathrm{C}$ is low.

Interestingly, the serum uromodulin concentration is positively correlated with GFR whereas conventional kidney function markers, such as creatinine, cystatin $\mathrm{C}$ and blood urea nitrogen, are negatively correlated with GFR. Moreover, the serum uromodulin concentration has a linear regression relationship with eGFR values. Intuitively, it would seem more advantageous to use a number with a positive correlation and linear regression relationship than a number with an inverse correlation and nonlinear regression relationship.

Given the difficulties involved in evaluating residual kidney function in patients on maintenance dialysis, their data were excluded from the scatter diagram in this study. However, there is no doubt that the GFR is $<5 \mathrm{~mL} / \mathrm{min}$ in these patients. In the present study, the mean eGFR-cre value in our patients on maintenance dialysis was $4.2 \mathrm{~mL} / \mathrm{min} / 1.73 \mathrm{~m}^{2}$. Taking into account the error in the eGFR value itself and the patterns of uromodulin concentration, that is, high in subjects with good kidney function and low in those with decreased kidney function, it might not necessarily be a mistake to incorporate data for patients on maintenance dialysis into a scatter plot. When these data were included, the correlation coefficient for serum uromodulin concentration and the eGFR value naturally increased (data not shown).

Uromodulin is a protein derived from renal tubular cells. Although most of the uromodulin is excreted into the urine, a very small amount of uromodulin that is expressed on the basolateral side of the tubular cells is not cleared and enters the circulation via blood vessels in the interstitial area. Thornley et al. reported that urinary excretion of uromodulin decreases in patients with decreased kidney function and tubular damage, and suggested that the serum uromodulin concentration decreases with worsening CKD stage because of a decreased expression level in the renal tubules [17].

Serum uromodulin could be a useful biomarker for evaluation of kidney functional reserve in all stages of CKD. However, little is known about the factors that regulate its protein expression and affect uromodulin concentrations. Furthermore, it is not known if renal and serum uromodulin protein expression levels show individual or racial differences. Given that no standard reference protein for uromodulin measurement has been established, we cannot directly compare our data with those in the past reports. Nonetheless, similar results have been reported using other ELISA kits or method [3-5, 17], and suggest that there is a certain value in the usefulness of uromodulin measurement. 
Analysis of mean serum uromodulin concentrations using the eGFR-cre value-based staging system, a significant decrease in serum uromodulin concentration was observed below an eGFR-cre value of 60 $\mathrm{mL} / \mathrm{min} / 1.73 \mathrm{~m}^{2}$ (Figure $2 \mathrm{~A}$ ). On analysis of mean serum uromodulin concentrations using the eGFR-cys value-based system, a significant decrease in serum uromodulin concentration was observed in 60-89 $\mathrm{mL} / \mathrm{min} / 1.73 \mathrm{~m}^{2}$ of eGFR-cys (Figure $2 \mathrm{~B}$ ). Furthermore, when the classification according to the eGFR-cys value was subdivided and analyzed, a significant decrease in the serum uromodulin concentration was observed below eGFR-cys $90 \mathrm{~mL} / \mathrm{min} / 1.73 \mathrm{~m}^{2}$ (Figure 2C). These results suggest that early decline of kidney function could be detected by serum uromodulin measurement as in previous reports [3-5].

When we evaluated the scatter plot as a whole, there was a strong positive correlation between the serum uromodulin concentration and the eGFR value but there is also considerable variation in the serum uromodulin concentration in the population with relatively good kidney function, that is, an eGFR $>60$ $\mathrm{mL} / \mathrm{min} / 1.73 \mathrm{~m}^{2}$. This trend is similar to the past reports [3-5]. Thornley et al. found a good correlation between serum uromodulin concentration and kidney function in patients with CKD but not in normal subjects (17). Risch et al. investigated the serum uromodulin concentration in patients with good kidney function, whose average eGFR-cys value was $85 \mathrm{~mL} / \mathrm{min} / 1.73 \mathrm{~m}^{2}$, and found only a weak correlation of $r$ $=0.38[24]$.

It is widely known that the neonatal glomerulus is immature and that the GFR at birth is one-fourth to onefifth that of an adult. Furthermore, the nephron number in an adult has been estimated to be one million per kidney; however, recent studies have shown that the nephron number in a Japanese adult is about 0.7 million per kidney [25] and that the glomerular density in a low-birth-weight infant is significantly low [26]. These reports suggest that there may be racial and individual differences in both the nephron number and their development. However, because the glomerulus is basically equipped with a hyperfiltration mechanism, a normal glomerulus can perform more than twice as much work as usual. Therefore, a slight variation in nephron number is unlikely to affect the GFR value. Furthermore, uromodulin is a tubule-derived protein and is not filtered by the glomeruli because of its high molecular weight. Variation of serum uromodulin concentration in groups with relatively good kidney function may reflect the number of functioning nephrons.

The eGFR value is an estimate of kidney function and also indicates the current "residual" kidney function. Looking at uromodulin concentration from the perspective of evaluating residual kidney function, we believe that uromodulin can be used not only as a biomarker for predicting kidney function but also as a novel tool in the field of preventive medicine. It should be possible to measure uromodulin levels at a young age, when kidney function is normal and before the onset of conditions such hypertension, diabetes, and chronic glomerulonephritis, all of which increase the risk of renal impairment. It is still unclear whether individuals with a high uromodulin concentration have high renal reserve and those with a low uromodulin concentration have poor renal reserve. However, there are two case reports on patients who were found to have a decreased uromodulin concentration related to allograft failure and 
graft loss after kidney transplantation $[27,28]$. These reports suggest that the higher the uromodulin concentration, the greater the renal reserve.

This study has some limitations, the main one being that we compared only the serum uromodulin concentration with the eGFR value and did not include an inulin clearance test. The eGFR is only an estimated value and does not necessarily guarantee equivalence with the inulin clearance value; however, evaluation of kidney function based on the eGFR value is well accepted in daily clinical practice. We evaluated the relationship between the uromodulin concentration and the eGFR-cre and eGFR-cys values and found that both were highly correlated.

\section{Conclusion}

In conclusion, we have obtained mAbs and established an ELISA kit for measuring the blood uromodulin concentration. A clinical study using this kit confirmed a good correlation between the serum uromodulin concentration and kidney function in a Japanese population. Furthermore, this study has demonstrated the usefulness of the uromodulin measurement in detection of an early decline in kidney function that cannot be detected by measurement of creatinine.

\section{Abbreviations}

BMI: body mass index; BSA: body surface area; CKD: chronic kidney disease; CV: coefficients of variation; dBP: diastolic blood pressure; eGFR: estimated glomerular filtration rate; eGFR-cre: creatinine-calculated eGFR; eGFR-cys: cystatin C-calculated eGFR; ELISA: enzyme-linked immunosorbent assay; HRP: horseradish peroxidase; HV: healthy volunteer; IQR: interquartile range; mAb: monoclonal antibody; PCR: polymerase chain reaction; SBP: systolic blood pressure; SD: standard deviation; SDS-PAGE: sodium dodecyl sulfate polyacrylamide gel electrophoresis

\section{Declarations}

\section{Acknowledgments}

The authors thank Drs Mio Kodama, Yohei Tsuchiya, and Maiko Hori for their assistance with recruitment of patients for this study and collection of clinical data and blood samples.

\section{Author's contributions}

R.U. contributed to conception and design of this study, analyzing data, writing the first draft and having final responsibility for the decision to submit for publication. T.O. and K.N. contributed to giving advice for study design and revising manuscript. R.U., H.T., C.I., M.K., T.M., M.H. contributed to enrolling study participants and acquisition of data. All authors reviewed and approved the manuscript. 


\section{Funding}

None.

\section{Availability of data and materials}

The datasets used and/or analyzed during the current study are available from the corresponding author on reasonable request.

\section{Ethics approval and consent to participate}

This study was approved by the ethics committees of each participating medical institution, such as Tokyo Women's Medical University (approval number; 4602), Minami Akatsuka Clinic and Yokohama Rosai Hospital, and conducted in adherence with the Declaration of Helsinki. All participants provided written informed consent prior to study enrollment.

\section{Consent for publication}

Not applicable.

\section{Competing interests}

The authors declare that they have no competing interests.

\section{Author details}

${ }^{1}$ Division of Nephrology, Department of Medicine, Yachiyo Medical Center, Tokyo Women's Medical University, Chiba, Japan., ${ }^{2}$ Department of Medicine, Tokyo Women's Medical University Medical Center East, Tokyo, Japan. ${ }^{3}$ Minami Akatsuka Clinic, Ibaraki, Japan. ${ }^{4}$ Department of Medicine, Division of Nephrology, Yokohama Rosai Hospital, Kanagawa, Japan. ${ }^{5}$ Department of Medicine, Kidney Center, Tokyo Women's Medical University, Tokyo, Japan.

\section{References}


1. Tamm I, Horsfall FL Jr. Characterization and separation of an inhibitor of viral hemagglutination present in urine. Proc Soc Exp Biol Med. 1950; 74: 106-108

2. Sarnak MJ, Levey AS, Schoolwerth AC, Coresh J, Culleton B, Hamm LL, et al. American Heart Association Councils on Kidney in Cardiovascular Disease, High Blood Pressure Research, Clinical Cardiology, and Epidemiology and Prevention. Kidney disease as a risk factor for development of cardiovascular disease: a statement from the American Heart Association Councils on Kidney in Cardiovascular Disease, High Blood Pressure Research, Clinical Cardiology, and Epidemiology and Prevention. Circulation. 2003; 108: 2154-2169

3. Steubl D, Block M, Herbst V, Nockher WA, Schlumberger W, Satanovskij R, et al. Plasma Uromodulin Correlates With Kidney Function and Identifies Early Stages in Chronic Kidney Disease Patients. Medicine. 2016; 95: e3011

4. Fedak D, Kuźniewski M, Fugiel A, Wieczorek-Surdacka E, Przepiórkowska-Hoyer B, et al. Serum uromodulin concentrations correlate with glomerular filtration rate in patients with chronic kidney disease. Pol Arch Med Wewn. 2016; 126: 995-1004

5. Scherberich JE, Gruber R, Nockher WA, Christensen El, Schmitt H, Herbst V, et al. Serum uromodulin-a marker of kidney function and renal parenchymal integrity. Nephrol Dial Transplant. 2018; 33: 284295

6. Köttgen A, Glazer NL, Dehghan A, Hwang SJ, Katz R, Li M, et al. Multiple loci associated with indices of renal function and chronic kidney disease. Nat Genet. 2009; 41: 712-717

7. Köttgen A, Hwang SJ, Larson MG, Van Eyk JE, Fu Q, Benjamin EJ, et al. Uromodulin levels associate with a common UMOD variant and risk for incident CKD. J Am Soc Nephrol. 2010; 21: 337-344

8. Gorski M, Tin A, Garnaas M, McMahon GM, Chu AY, Tayo BO, et al. Genome-wide association study of kidney function decline in individuals of European descent. Kidney Int. 2015; 87: 1017-1029

9. Trudu M, Janas S, Lanzani C, Debaix H, Schaeffer C, Ikehata M, et al. Common noncoding UMOD gene variants induce salt-sensitive hypertension and kidney damage by increasing uromodulin expression. Nat Med. 2013; 19: 1655-1660

10. Leiherer A, Muendlein A, Saely CH, Ebner J, Brandtner EM, Fraunberger P, et al. Serum uromodulin is a predictive biomarker for cardiovascular events and overall mortality in coronary patients. Int $\mathrm{J}$ Cardiol. 2017; 231; 6-12

11. Delgado GE, Kleber ME, Scharnagl H, Krämer BK, März W, Scherberich JE. Serum Uromodulin and Mortality Risk in Patients Undergoing Coronary Angiography. J Am Soc Nephrol. 2017; 28: 2201-2210

12. El-Achkar TM, Wu XR, Rauchman M, McCracken R, Kiefer S, Dagher PC. Tamm-Horsfall protein protects the kidney from ischemic injury by decreasing inflammation and altering TLR4 expression. Am J Physiol Renal Physiol. 2008; 295: F534-44

13. El-Achkar TM, McCracken R, Rauchman M, Heitmeier MR, Al-Aly Z, Dagher PC, et al. Tamm-Horsfall protein-deficient thick ascending limbs promote injury to neighboring S3 segments in an MIP-2dependent mechanism. Am J Physiol Renal Physiol. 2011; 300: F999-F1007 
14. Matsuo S, Imai E, Horio M, Yasuda Y, Tomita K, Nitta K, et al. Revised equations for estimated GFR from serum creatinine in Japan. Collaborators developing the Japanese equation for estimated GFR. Am J Kidney Dis. 2009; 53: 982-992

15. Horio M, Imai E, Yasuda Y, Watanabe T, Matsuo S. GFR estimation using standardized serum cystatin $C$ in Japan. Collaborators Developing the Japanese Equation for Estimated GFR. Am J Kidney Dis. 2013; 61: 197-203

16. Dawnay AB, Cattell WR. Serum Tamm-Horsfall glycoprotein levels in health and in renal disease. Clin Nephrol. 1981; 15: 5-8

17. Thornley C, Dawnay A, Cattell WR. Human Tamm-Horsfall glycoprotein: urinary and plasma levels in normal subjects and patients with renal disease determined by a fully validated radioimmunoassay. Clin Sci. 1985; 68: 529-535

18. Prajczer S, Heidenreich U, Pfaller W, Kotanko P, Lhotta K, Jennings P. Evidence for a role of uromodulin in chronic kidney disease progression. Nephrol Dial Transplant. 2010; 25: 1896-1903

19. Levey AS, Coresh J, Greene T, Marsh J, Stevens LA, Kusek JW, et al. Expressing the Modification of Diet in Renal Disease Study equation for estimating glomerular filtration rate with standardized serum creatinine values. Clin Chem. 2007; 53: 766-772

20. Inker LA, Schmid CH, Tighiouart $\mathrm{H}$, Eckfeldt JH, Feldman $\mathrm{HI}$, Greene $\mathrm{T}$, et al. Estimating glomerular filtration rate from serum creatinine and cystatin C. N Engl J Med. 2012; 367: 20-29

21. Jayagopal V, Keevil BG, Atkin SL, Jennings PE, Kilpatrick ES. Paradoxical changes in cystatin $C$ and serum creatinine in patients with hypo- and hyperthyroidism. Clin Chem. 2003; 49: 680-681

22. Cimerman N, Brguljan PM, Krasovec M, Suskovic S, Kos J. Serum cystatin C, a potent inhibitor of cysteine proteinases, is elevated in asthmatic patients. Clin Chim Acta. 2000; 300: 83-95

23. Kazama JJ, Kutsuwada K, Ataka K, Maruyama H, Gejyo F. Serum cystatin C reliably detects renal dysfunction in patients with various renal diseases. Nephron. 2002; 91: 13-20

24. Risch L, Lhotta K, Meier D, Medina-Escobar P, Nydegger UE, Risch M. The serum uromodulin level is associated with kidney function. Clin Chem Lab Med. 2014; 52: 1755-1761

25. Sasaki T, Tsuboi N, Kanzaki G, Haruhara K, Okabayashi Y, Koike K, Kobayashi A, Yamamoto I, Ogura M, Hoy WE, Bertram JF, Shimizu A, Yokoo T. Biopsy-based estimation of total nephron number in Japanese living kidney donors. Clin Exp Nephrol. 2019; 23: 629-637

26. Koike K, Ikezumi Y, Tsuboi N, Kanzaki G, Haruhara K, Okabayashi Y, Sasaki T, Ogura M, Saitoh A, Yokoo T. Glomerular Density and Volume in Renal Biopsy Specimens of Children with Proteinuria Relative to Preterm Birth and Gestational Age. Clin J Am Soc Nephrol. 2017; 12: 585-590

27. Steubl D, Block M, Herbst V, Schlumberger W, Nockher A, Angermann S, Schmaderer C, Heemann U, Renders $\mathrm{L}$, Scherberich J. Serum uromodulin predicts graft failure in renal transplant recipients. Biomarkers. 2017; 22: 171-177

28. Bostom A, Steubl D, Garimella PS, Franceschini N, Roberts MB, Pasch A, Ix JH, Tuttle KR, Ivanova A, Shireman T, Kim SJ, Gohh R, Weiner DE, Levey AS, Hsu CY, Kusek JW, Eaton CB. Serum Uromodulin: A Biomarker of Long-Term Kidney Allograft Failure Am J Nephrol. 2018; 47: 275-282 


\section{Tables}

Due to technical limitations, the tables are only available as a download in the supplemental files section.

Table 1. Characteristics of the study participants according to sex

The data are presented as the mean (with the standard deviation), the median and interquartile range, or as the number and percentage. BMI, body mass index; BSA, body surface area; SBP, systolic blood pressure; dBP, diastolic blood pressure; eGFR-cre, estimated glomerular filtration rate calculated from creatinine; eGFR-cys, eGFR calculated from cystatin C.

Table 2. Characteristics of patients with and without CKD and of healthy volunteers

The data are presented as the mean and standard deviation, median (interquartile range), or number (percentage). HV, healthy volunteer; CKD, chronic kidney disease

\section{Figures}



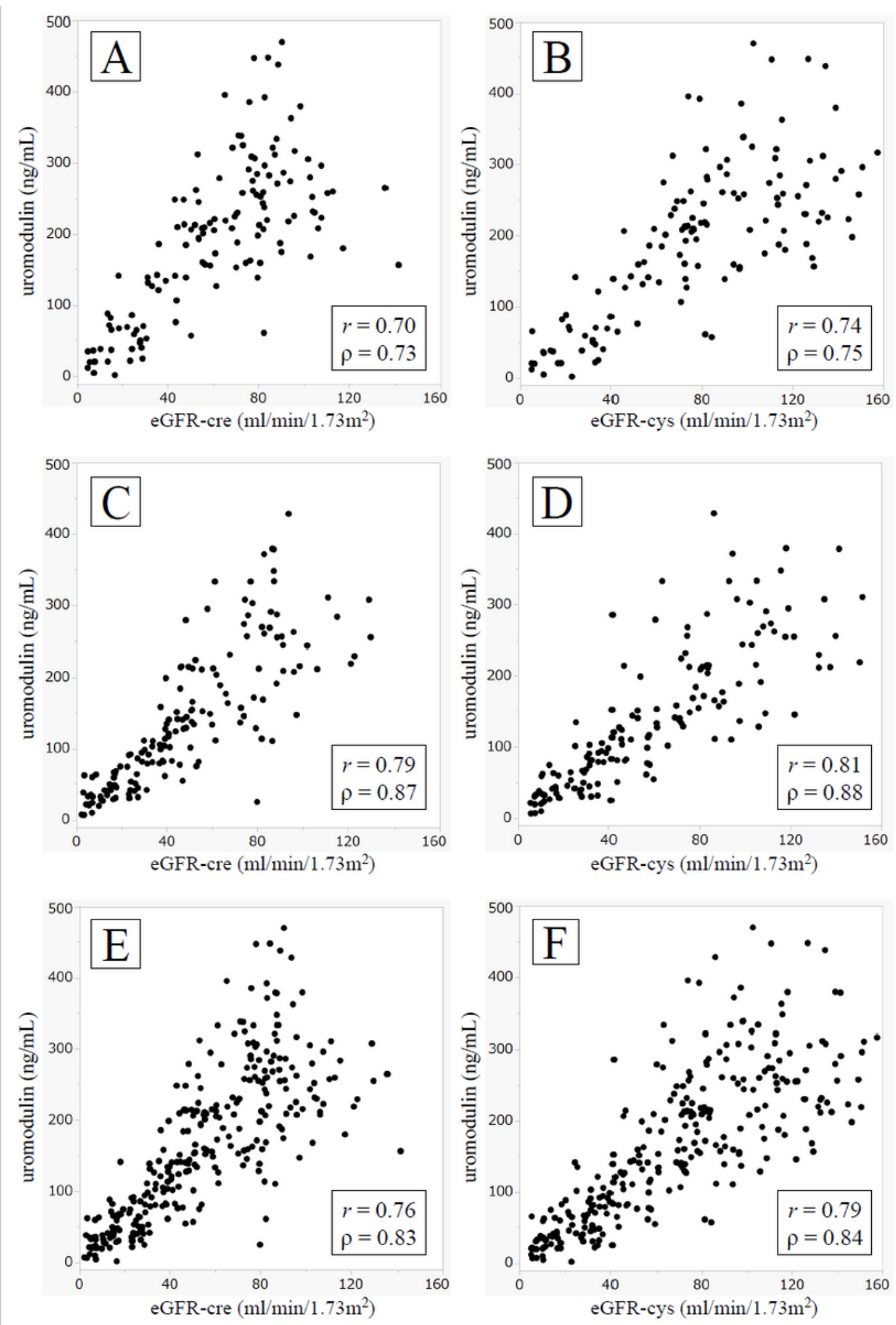

\section{Figure 1}

Correlations between serum uromodulin concentration and eGFR Scatter diagrams showing the serum uromodulin concentration and eGFR values calculated from serum creatinine by sex and in both sexes (A, female; $C$, male; $E$, both sexes), and serum cystatin $C$ (B, female; $D$, male; $F$, both sexes). The data for patients on hemodialysis were excluded. r, Pearson's product moment correlation coefficient; $\mathrm{P}$, Spearman's rank correlation coefficient. 

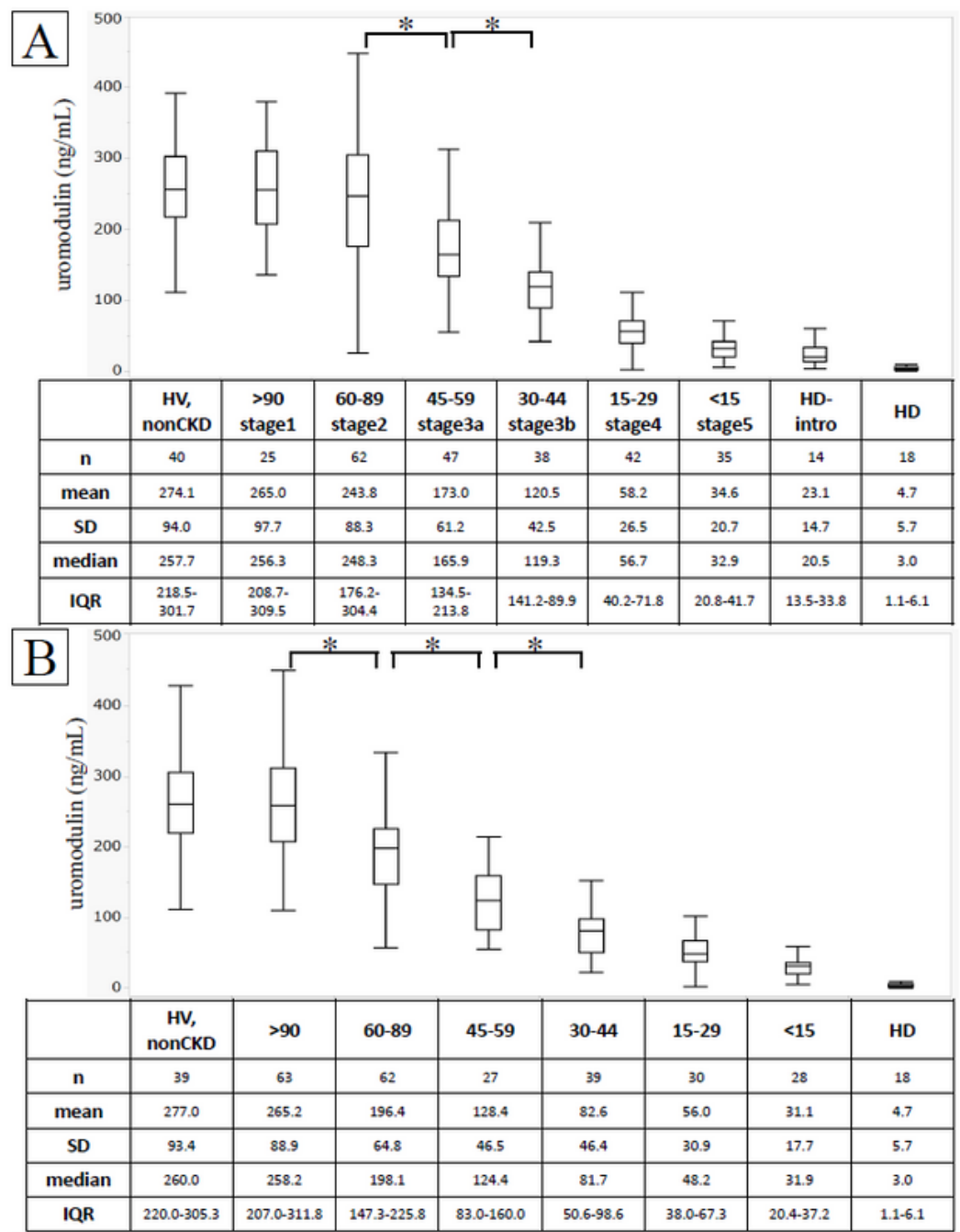

$\mathrm{C}$

\begin{tabular}{|c|c|c|c|c|c|}
\hline & HV,nonCKD & $>90$ & $81-90$ & $71-80$ & $61-70$ \\
\hline$n$ & ${ }^{43}$ & 60 & ${ }^{21}$ & ${ }^{28}$ & 14 \\
\hline mean & 274.0 & 266.8 & 205.9 & 190.3 & 195.8 \\
\hline SD & 92.1 & 90.3 & 67.8 & 58.2 & 73.6 \\
\hline median & 260.0 & 258.7 & 217.7 & 188.7 & 171.8 \\
\hline IQR & 5.3 & 2073.3135 .5 & 168.8 .253 .2 & 6 & 1345.250 .2 \\
\hline
\end{tabular}

\section{Figure 2}

Box plot analysis Box plots showing the serum uromodulin concentration in patients with CKD stages 1 to 5 , those on chronic maintenance hemodialysis, patients without CKD, and healthy volunteers at the time of introduction of hemodialysis. (A) Classification by the eGFR-cre value. $(B, C)$ Classification by the eGFR-cys value. *P <0.05. HV, healthy volunteer; CKD, chronic kidney disease; SD, standard deviation; IQR, interquartile range 


\section{Supplementary Files}

This is a list of supplementary files associated with this preprint. Click to download.

- SupplementaryfigureS3.pdf

- SupplementaryfigureS4.pdf

- SupplementaryfigureS1.pdf

- SupplementaryTables2.pdf

- SupplementaryTables3.pdf

- Table1.pdf

- SupplementaryTables1.pdf

- Supplementaryfigures2.pdf

- uromodulinusuiBMCsup.docx

- Table2.pdf 